\title{
Boundedness of One-Sided Oscillatory Integral Operators on Weighted Lebesgue Spaces
}

\author{
Zunwei Fu, ${ }^{1}$ Shanzhen Lu, ${ }^{2}$ Yibiao Pan, ${ }^{3}$ and Shaoguang Shi ${ }^{1}$ \\ ${ }^{1}$ Department of Mathematics, Linyi University, Linyi 276005, China \\ ${ }^{2}$ School of Mathematical Sciences, Beijing Normal University, Beijing 100875, China \\ ${ }^{3}$ Department of Mathematics, University of Pittsburgh, Pittsburgh, PA 15260, USA
}

Correspondence should be addressed to Shaoguang Shi; shishaoguang@mail.bnu.edu.cn

Received 6 October 2013; Accepted 9 December 2013; Published 3 February 2014

Academic Editor: S. A. Mohiuddine

Copyright (C) 2014 Zunwei Fu et al. This is an open access article distributed under the Creative Commons Attribution License, which permits unrestricted use, distribution, and reproduction in any medium, provided the original work is properly cited.

We consider one-sided weight classes of Muckenhoupt type, but larger than the classical Muckenhoupt classes, and study the boundedness of one-sided oscillatory integral operators on weighted Lebesgue spaces using interpolation of operators with change of measures.

\section{Introduction and Main Results}

Oscillatory integrals in one form or another have been an essential part of harmonic analysis from the very beginnings of that subject; three chapters are devoted to them in the celebrated Stein's book [1]. Many operators in harmonic analysis or partial differential equations are related to some versions of oscillatory integrals, such as the Fourier transform, the Bochner-Riesz means, and the Radon transform which has important applications in the CT technology. Among numerous papers dealing with oscillatory singular integral operators in some function spaces, we refer to [27] and the references therein. More generally, let us now consider a class of oscillatory integrals defined by Ricci and Stein $[8]$ :

$$
T f(x)=\text { p.v. } \int_{\mathbb{R}} e^{i P(x, y)} K(x-y) f(y) d y,
$$

where $P(x, y)$ is a real-valued polynomial defined on $\mathbb{R} \times \mathbb{R}$ and the function $K \in C^{1}(\mathbb{R} \backslash\{0\})$ is a Calderón-Zygmund kernel. That means $K$ satisfies

$$
\begin{gathered}
|K(x)| \leq \frac{C}{|x|}, \quad|\nabla K(x)| \leq \frac{C}{|x|^{2}}, \quad x \neq 0, \\
\int_{a<|x|<b} K(x) d x=0 \quad \forall a, b \quad(0<a<b) .
\end{gathered}
$$

Throughout this paper, the letter $C$ will denote a positive constant which may vary from line to line but will remain independent of the relevant quantities.

We state a celebrated result of Ricci and Stein on oscillatory integrals as follows.

Theorem 1 (see [8]). Let $1<p<\infty, K$ satisfy (2) and (3). Then for any real-valued polynomial $P(x, y)$, the oscillatory integral operator $T$ is of type $\left(L^{p}, L^{p}\right)$ and its norm depends on the total degree of $P$, but not on the coefficients of $P$ in other respects.

Weighted inequalities arise naturally in harmonic analysis, but their use is best justified by the variety of applications in which they appear. It is worth pointing out that many authors are interested in the inequalities when the weight functions belong to the Muckenhoupt classes ([9]), which are denoted by $A_{p}(1<p<\infty)$ classes for simplicity. This class consists of positive locally integrable functions (weight functions) $w$ for which

$$
\sup _{I}\left(\frac{1}{|I|} \int_{I} w(x) d x\right)\left(\frac{1}{|I|} \int_{I} w(x)^{1-p^{\prime}} d x\right)^{p-1}<\infty,
$$

where the supremum is taken over all intervals $I \subset \mathbb{R}$ and $1 / p+1 / p^{\prime}=1$ 
In 1992, Lu and Zhang [10] established the weighted version of Theorem 1 .

Theorem 2. Let $p, P(x, y)$ and $K$ be as in Theorem 1. Then the oscillatory singular integral operator $T$ is of type $\left(L^{p}(w)\right.$, $\left.L^{p}(w)\right)$ with $w \in A_{p}$. Here its operator norm is bounded by a constant depending on the total degree of $P$, but not on the coefficients of $P$ in other respects.

We point out that Theorems 1 and 2 also hold for dimension $n \geq 2$. We choose the results for $n=1$ here in order to introduce the one-sided operators which were defined on $\mathbb{R}$. Theorems 1 and 2 are also true for more general kernels, that is, nonconvolution kernels, under the $L^{2}$ boundedness assumption on the corresponding CalderónZygmund singular integral operators:

$$
\widetilde{T}(x)=\text { p.v. } \int_{\mathbb{R}^{n}} K(x-y) f(y) d y .
$$

However, this topic exceeds the scope of this paper. For more information about this work, see $[8,10]$, for example.

The study of weights for one-sided operators was motivated not only by the generalization of the theory of bothsided ones, but also by their natural appearance in harmonic analysis; for example, they are required when we treat the one-sided Hardy-Littlewood maximal operator [11]:

$$
\begin{aligned}
& M^{+} f(x)=\sup _{h>0} \frac{1}{h} \int_{x}^{x+h}|f(y)| d y, \\
& M^{-} f(x)=\sup _{h>0} \frac{1}{h} \int_{x-h}^{x}|f(y)| d y,
\end{aligned}
$$

arising in the ergodic maximal function. Sawyer first introduced the classical one-sided weight $A_{p}^{+}$classes in [11]. The general definitions of $A_{p}^{+}$and $A_{p}^{-}$were introduced in [12] as

$$
\begin{aligned}
& A_{p}^{+}: \sup _{a<b<c} \frac{1}{(c-a)^{p}} \int_{a}^{b} w(x) d x\left(\int_{b}^{c} w(x)^{1-p^{\prime}} d x\right)^{p-1} \leq C, \\
& A_{p}^{-}: \sup _{a<b<c} \frac{1}{(c-a)^{p}} \int_{b}^{c} w(x) d x\left(\int_{a}^{b} w(x)^{1-p^{\prime}} d x\right)^{p-1} \leq C,
\end{aligned}
$$

where $1<p<\infty, 1 / p+1 / p^{\prime}=1$; also, for $p=1$,

$$
A_{1}^{+}: M^{-} w \leq C w, \quad A_{1}^{-}: M^{+} w \leq C w .
$$

The smallest constant $C$ for which the above inequalities are satisfied will be denoted by $A_{p}^{+}(w)$ and $A_{p}^{-}(w), p \geq 1$. $A_{p}^{+}(w)\left(A_{p}^{-}(w)\right)$ will be called the $A_{p}^{+}$(resp., $A_{p}^{-}$) constant of $w$. By Lebesgue's differentiation theorem, we can easily prove $A_{1}^{+}(w)$ (resp., $\left.A_{1}^{-}(w)\right) \geq 1$. In [13], the class $A_{\infty}^{+}$was introduced as $A_{\infty}^{+}=\bigcup_{p<\infty} A_{p}^{+}$(see also [14]). It is easy to see that for $1 \leq p \leq \infty, A_{p} \subset A_{p}^{+}, A_{p} \subset A_{p}^{-}$, and $A_{p}=A_{p}^{+} \cap A_{p}^{-}$.

Theorem 3 (see [11]). Let $1<p<\infty$. Then

(1) $M^{+}$is bounded in $L^{p}(w)$ if and only if $w \in A_{p}^{+}$;

(2) $M^{-}$is bounded in $L^{p}(w)$ if and only if $w \in A_{p}^{-}$.
The one-sided weight classes are of interest, not only because they control the boundedness of the one-sided Hardy-Littlewood maximal operator, but also because they are the right classes for the weighted estimates of one-sided Calderón-Zygmund singular integral operators [15], which are defined by

$$
\begin{aligned}
& \widetilde{T}^{+} f(x)=\lim _{\varepsilon \rightarrow 0^{+}} \int_{x+\varepsilon}^{\infty} K(x-y) f(y) d y, \\
& \widetilde{T}^{-} f(x)=\lim _{\varepsilon \rightarrow 0^{+}} \int_{-\infty}^{x-\varepsilon} K(x-y) f(y) d y,
\end{aligned}
$$

where $K$ is the one-sided Calderón-Zygmund kernel with support in $\mathbb{R}^{-}=(-\infty, 0)$ and $\mathbb{R}^{+}=(0,+\infty)$, respectively. We say a function $K$ is a one-sided Calderón-Zygmund kernel if $K$ satisfies (2) and

$$
\left|\int_{a<|x|<b} K(x) d x\right| \leq C, \quad 0<a<b
$$

with support in $\mathbb{R}^{-}=(-\infty, 0)$ or $\mathbb{R}^{+}=(0,+\infty)$. An example of such a kernel is

$$
K(x)=\frac{\sin (\log |x|)}{(x \log |x|)} \chi_{(-\infty, 0)}(x),
$$

where $\chi_{E}$ denotes the characteristic function of a set $E$.

Theorem 4 (see [15]). Let $1<p<\infty$ and $K$ be a one-sided Calderón-Zygmund kernel. Then

(1) $\widetilde{T}^{+}$is bounded in $L^{p}(w)$ if and only if $w \in A_{p}^{+}$;

(2) $\widetilde{T}^{-}$is bounded in $L^{p}(w)$ if and only if $w \in A_{p}^{-}$.

Theorem 4 is the one-sided version of weighted norm inequality of singular integral due to Coifman and Fefferman [9].

Highly inspired by the above statements for oscillatory singular integral operators and one-sided operator theory, in [16], the authors had introduced the one-sided oscillatory singular integral operators and studied the weighted weak type $(1,1)$ norm inequalities for these operators. In this paper, we will further study the one-sided Muckenhoupt weight classes and give the one-sided version of Theorem 2. It is well known that the property of the one-sided Muckenhoupt weight classes is worse than the Muckenhoupt weight classes (see also [17]). For example, both the reverse Hölder inequality and the doubling condition are not true for the one-sided case. Therefore, some new methods are needed to deal with some new difficulties.

We first recall the definition of one-sided oscillatory integral operator as

$$
\begin{aligned}
& T^{+} f(x)=\lim _{\varepsilon \rightarrow 0^{+}} \int_{x+\varepsilon}^{\infty} e^{i P(x, y)} K(x-y) f(y) d y, \\
& T^{-} f(x)=\lim _{\varepsilon \rightarrow 0^{+}} \int_{-\infty}^{x-\varepsilon} e^{i P(x, y)} K(x-y) f(y) d y,
\end{aligned}
$$

where $P(x, y)$ is a real-valued polynomial defined on $\mathbb{R} \times \mathbb{R}$ and the kernel $K$ is a one-sided Calderón-Zygmund kernel 
with support in $\mathbb{R}^{-}$and $\mathbb{R}^{+}$, respectively. Now, we formulate our results as follows.

Theorem 5. Let $1<p<\infty$ and $K$ be a one-sided CalderónZygmund kernel. Then for any real-valued polynomial $P(x, y)$,

(1) there exists constant $C>0$ such that

$$
\left\|T^{+} f\right\|_{L^{p}(w)} \leq C\|f\|_{L^{p}(w)},
$$

where $w \in A_{p}^{+}$and the operator norm depend on the total degree of $P$ and $A_{p}^{+}(w)$, but not on the coefficients of $P$ in other respects;

(2) there exists constant $C>0$ such that

$$
\left\|T^{-} f\right\|_{L^{p}(w)} \leq C\|f\|_{L^{p}(w)}
$$

where $w \in A_{p}^{-}$and the operator norm depend on the total degree of $P$ and $A_{p}^{-}(w)$, but not on the coefficients of $P$ in other respects.

The rest of this paper is devoted to the argument for Theorem 5. Section 2 contains some preliminaries which are essential to our proof. In Section 3, we will give the proof of Theorem 5.

\section{Preliminaries}

Lemma 6 (see $[11,18]$ ). Let $1<p<\infty$ and $w \geq 0$ be locally integrable. Then the following statements are equivalent:

(1) $w \in A_{p}^{+}$;

(2) $w^{1-p^{\prime}} \in A_{p^{\prime}}^{-}$;

(3) there exist $w_{1} \in A_{1}^{+}$and $w_{2} \in A_{1}^{-}$such that $w=$ $w_{1}\left(w_{2}\right)^{1-p}$.

According to the definition of $A_{p}^{+}$, we can easily obtain the following lemma.

Lemma 7. Let $1<p<\infty$ and $w \in A_{p}^{+}$. Then $A_{p}^{+}\left(\delta^{\lambda}(w)\right)=$ $A_{p}^{+}(w)$, where $\delta^{\lambda}(w)(x)=w(\lambda x)$ for all $\lambda>0$.

Proof. For $1<p<\infty$, if $w \in A_{p}^{+}$, then

$$
\sup _{a<b<c} \frac{1}{(c-a)^{p}} \int_{a}^{b} w(x) d x\left(\int_{b}^{c} w(x)^{1-p^{\prime}} d x\right)^{p-1} \leq C .
$$

For $\lambda>0, a^{\prime}=\lambda a, b^{\prime}=\lambda b, c^{\prime}=\lambda c$, and $d^{\prime}=\lambda d$, we have

$$
\begin{aligned}
& \frac{1}{(c-a)^{p}} \int_{a}^{b} w(\lambda x) d x\left(\int_{b}^{c} w(\lambda x)^{1-p^{\prime}} d x\right)^{p-1} \\
& =\frac{1}{(c-a)^{p}} \int_{a \lambda}^{b \lambda} w(x) \lambda^{-1} d x\left(\int_{b \lambda}^{c \lambda} w(x)^{1-p^{\prime}} \lambda^{-1} d x\right)^{p-1} \\
& =\frac{1}{(\lambda(c-a))^{p}} \int_{a \lambda}^{b \lambda} w(x) d x\left(\int_{b \lambda}^{c \lambda} w(x)^{1-p^{\prime}} d x\right)^{p-1} \\
& =\frac{1}{\left(c^{\prime}-a^{\prime}\right)^{p}} \int_{a^{\prime}}^{b^{\prime}} w(x) d x\left(\int_{b^{\prime}}^{c^{\prime}} w(x)^{1-p^{\prime}} d x\right)^{p-1}
\end{aligned}
$$$$
\leq C \text {. }
$$

The proof is complete.

We say a weight $w$ satisfies the one-sided reverse Hölder $R H_{r}^{+}$condition [18] if there exists $C>0$ such that for any $a<b$ and $1<r<\infty$,

$$
\int_{a}^{b} w(x)^{r} d x \leq C\left(M\left(w \chi_{(a, b)}\right)(b)\right)^{r-1} \int_{a}^{b} w(x) d x
$$

where $M$ is the classical Hardy-Littlewood maximal operator. The smallest such constant will be called the $\mathrm{RH}_{r}^{+}$constant of $w$ and will be denoted by $\mathrm{RH}_{r}^{+}(w)$. Corresponding to the classical reverse Hölder inequality, (17) is named the weak reverse Hölder inequality. For $r=\infty$, we say a weight $w$ satisfies the one-sided reverse Hölder $\mathrm{RH}_{\infty}^{+}$condition if there exists $C>0$ such that $w(x) \leq C m^{+} w(x)$ for almost all $x \in \mathbb{R}$ where $\mathrm{m}^{+}$is the one-sided minimal operator defined as

$$
m^{+} f(x)=\inf _{h>0} \frac{1}{h} \int_{x}^{x+h}|f| d y .
$$

The smallest such constant will be called the $\mathrm{RH}_{\infty}^{+}$constant of $w$ and will be denoted by $R H_{\infty}^{+}(w)$. It is clear that $R H_{\infty}^{+}(w) \geq$ 1. In [18], the authors give several characterizations of $R_{r}^{+}$ where the constants $C$ are not necessary the same.

Lemma 8. Let $a<b<c<d, 1<r<\infty$, and $w \geq 0$ be locally integrable. Then the following statements are equivalent:

(1) $\int_{a}^{b} w(x)^{r} d x \leq C\left(M\left(w \chi_{(a, b)}\right)(b)\right)^{r-1} \int_{a}^{b} w(x) d x$;

(2) $(1 /(b-a)) \int_{a}^{b} w(x)^{r} d x \leq C\left((1 /(c-b)) \int_{b}^{c} w(x) d x\right)^{r}$ with $b-a=2(c-b)$;

(3) $(1 /(b-a)) \int_{a}^{b} w(x)^{r} d x \leq C\left((1 /(d-c)) \int_{c}^{d} w(x) d x\right)^{r}$ with $b-a=d-b=2(d-c)$;

(4) $(1 /(b-a)) \int_{a}^{b} w(x)^{r} d x \leq C\left((1 /(c-b)) \int_{b}^{c} w(x) d x\right)^{r}$ with $b-a=c-b$;

(5) $(1 /(b-a)) \int_{a}^{b} w(x)^{r} d x \leq C\left((1 /(d-c)) \int_{c}^{d} w(x) d x\right)^{r}$ with $b-a=d-c=\gamma(d-a), 0<\gamma \leq 1 / 2$. 
Lemma 9 (see [18]). A weight $w \in A_{p}^{+}$for $p>1$ if and only if there exist $0<\gamma<1 / 2$ and a constant $C_{\gamma}$ such that for $b-a=d-c=\gamma(d-a)$ with $a<b<c<d$, the following inequality holds:

$$
\int_{a}^{b} w(x) d x\left(\int_{c}^{d} w(x)^{1-p^{\prime}} d x\right)^{p-1} \leq C_{\gamma}(b-a)^{p} .
$$

Combining the results in $[12,15,18,19]$, we can deduce Lemma 10. In what follows, we will include its proof with slight modifications for the sake of completeness.

Lemma 10. Let $w \in A_{p}^{+}$. Then there exists $\varepsilon>0$ such that $w^{1+\varepsilon} \in A_{p}^{+}$.

Proof. By Lemma 6, we have $w=w_{1} w_{2}^{1-p}$ with $w_{1} \in A_{1}^{+}$ and $w_{2} \in A_{1}^{-}$. For fixed interval $I=(a, b)$, we next claim that $w_{1} \in R H_{r}^{+}$for all $1<r<C /(C-1)$ with $C=$ $\max \left\{A_{1}^{+}\left(w_{1}\right), A_{1}^{-}\left(w_{1}\right)\right\}>1$. In fact, we consider the truncation of $w$ at height $H$ defined by $w_{H}=\min \left\{w_{1}, H\right\}$ which also satisfies $A_{1}^{+}$condition (with a constant $C_{H} \leq C$ ). Therefore, if $\lambda_{I}=M\left(w_{H} \chi_{I}\right)(b)$ and $S_{\lambda}=\left\{x \in I: w_{H}(x)>\lambda\right\}$, then we have

$$
\int_{S_{\lambda}} w_{H}(x) d x \leq C_{H} \lambda\left|S_{\lambda}\right|, \quad \lambda \geq \lambda_{I} .
$$

Indeed, it is straightforward if $S_{\lambda}=I$ since

$$
w_{H}\left(S_{\lambda}\right)=\int_{a}^{b} w_{H}(x) d x \leq \lambda_{I}(b-a) \leq C_{H} \lambda\left|S_{\lambda}\right| .
$$

We now assume $S_{\lambda} \neq I$ and fix $\varepsilon>0$ and an open set $O$ such that $S_{\lambda} \subset O \subset I$ with $|O| \leq \varepsilon+\left|S_{\lambda}\right|$. Let $O_{i}=(c, d)$, which is connected. There are two cases; that is, $a \leq c<d<b$ and $a \leq c<d=b$. In the first case, it is easy to check that $d$ is not contained in $S_{\lambda}$. By the definition of $S_{\lambda}, w_{1}^{+}$, we have $\int_{c}^{d} w_{H}(x) d x \leq C_{H} \lambda(d-c)$, while the second case is handled as the case $S_{\lambda}=I$ since $\int_{c}^{d} w_{H}(x) d x \leq C(b-c)$. Thus $w_{H}\left(O_{i}\right) \leq C_{H} \lambda\left|O_{i}\right|$. Adding up with $i$, we get

$$
w_{H}\left(S_{\lambda}\right) \leq w_{H}(O) \leq C_{H} \lambda\left|O_{i}\right| \leq C_{H} \lambda\left(\varepsilon+\left|S_{\lambda}\right|\right) .
$$

Therefore, we obtain (20). For fixed $\theta>-1$, multiply both sides of (20) by $\lambda^{\theta}$ and integrate from $\lambda_{I}$ to infinity; we can obtain

$$
\frac{1}{\theta+1} \int_{I}\left(w_{H}^{\theta+2}-\lambda_{I}^{\theta+1}\right)(x) d x \leq \frac{C_{H}}{\theta+2} \int_{I} w_{H}^{\theta+2}(x) d x .
$$

Now if $r=\theta+2<C_{H} /\left(C_{H^{-}}-1\right)$, then $1 /(\theta+1)-C_{H} /(\theta+2)>0$, which implies

$$
\begin{aligned}
\int_{I} w_{H}^{r}(x) d x & \leq C_{H} \lambda_{I}^{r-1} \int_{I} w_{H}(x) d x \\
& =C_{H}\left(M\left(w_{H} \chi_{I}\right)(b)\right)^{r-1} \int_{I} w_{H}(x) d x .
\end{aligned}
$$

The inequality $C_{H} \leq C$ implies $C_{H} /\left(C_{H}-1\right) \geq C /(C-1)$. Therefore, if $r \leq C /(C-1)$, then we have

$$
\begin{aligned}
\int_{I} w_{H}^{r}(x) d x & =C_{H}\left(M\left(w_{H} \chi_{I}\right)(b)\right)^{r-1} \int_{a}^{b} w_{H}(x) d x \\
& =C\left(M\left(w_{1} \chi_{(a, b)}\right)(b)\right)^{r-1} \int_{a}^{b} w_{H}(x) d x .
\end{aligned}
$$

Hence $w_{1} \in R H_{r}^{+}$by the monotone convergence theorem. Since $w_{2} \in A_{1}^{-}$, we next claim that $w_{2}^{1-p} \in R H_{\infty}^{+}$. In fact, for any interval $I=(a, b)$, we have

$$
\left(\frac{1}{|I|} \int_{I} w_{2}(x) d x\right)^{1-p} \leq \frac{1}{|I|} \int_{I} w_{2}(x)^{1-p} d x
$$

by Hölder's inequality and the $A_{1}^{-}$condition. For almost every $x \in I^{-}=(2 a-b, a)$, we have

$$
C w_{2} \geq \frac{1}{|I|} \int_{I} w_{2}(x) d x
$$

Thus,

$$
\begin{aligned}
w_{2}(x)^{1-p} & \leq C\left(\frac{1}{|I|} \int_{I} w_{2}(x) d x\right)^{1-p} \\
& \leq C \frac{1}{|I|} \int_{I} w_{2}(x)^{1-p} d x \\
& \leq C \frac{1}{b-x} \int_{x}^{b} w_{2}(x)^{1-p} d x,
\end{aligned}
$$

which implies our claim. Hence,

$$
\begin{aligned}
\frac{1}{|I|} \int_{I} w^{r} & \leq \frac{1}{|I|} \int_{I} w_{1}^{r} \sup _{I}\left(w_{2}^{-r(p-1)}\right) \\
& \leq C\left(\frac{1}{I_{1}} \int_{I_{1}} w_{1}\right)^{r}\left(\frac{1}{I_{1}} \int_{I_{1}} w_{2}^{1-p}\right)^{r} \\
& \leq C\left(\inf _{I_{1}} w_{1}\right)^{r}\left(\sup _{I_{1}} w_{2}^{1-p}\right)^{r} \\
& \leq C\left(\inf w_{1}\right)^{r}\left(\frac{1}{I_{2}} \int_{I_{2}} w_{2}^{1-p}\right)^{r} \\
& \leq C\left(\frac{1}{I_{2}} \int_{I_{2}} w\right)^{r}
\end{aligned}
$$

where $I_{1}=(b, 2 b-a)$ and $I_{2}=(2 b-a, 3 b-2 a)$. By Lemma 8 , we obtain $w \in R H_{r}^{+}$. Hence, $w^{1-p^{\prime}} \in R H_{r}^{-}$for all $1<r<$ $C /(C-1)$ by Lemma 6.

Let us fix $a<d$ and choose $b, c$ such that $b-a=d-c=$ $(d-a) / 4$ (e.g., we choose $b=(d+3 a) / 4, c=(3 d+a) / 4)$. Following from the five points $a, b,(b+c) / 2, c, d$, we have four intervals, namely,

$$
\begin{aligned}
& I_{1}=(a, b), \quad I_{2}=\left(b, \frac{(b+c)}{2}\right), \\
& I_{3}=\left(\frac{(b+c)}{2}, c\right), \quad I_{4}=(c, d) .
\end{aligned}
$$


By Lemma 8, we have

$$
\begin{aligned}
& \frac{1}{\left|I_{1}\right|} \int_{I} w^{r}\left(\frac{1}{\left|I_{4}\right|} \int_{I_{4}} w^{r\left(1-p^{\prime}\right)}\right)^{p-1} \\
& \quad \leq\left(\frac{1}{\left|I_{2}\right|} \int_{I_{2}} w\right)^{r}\left(\frac{1}{\left|I_{3}\right|} \int_{I_{3}} w^{\left(1-p^{\prime}\right)}\right)^{r(p-1)} \leq C^{r} .
\end{aligned}
$$

Thus, $w^{r} \in A_{p}^{+}$by Lemma 9. Choosing $0<\varepsilon=r-1<1$ / $(C-1)$, then we complete the proof of the lemma.

To prove Theorem 5, we still need a celebrated interpolation theorem of operators with change of measures.

Lemma 11 (see [20]). Suppose that $u_{0}, v_{0}, u_{1}, v_{1}$ are positive weight functions and $1<p_{0}, p_{1}<\infty$. Assume sublinear operator $S$ satisfies

$$
\begin{aligned}
& \|S f\|_{L^{p_{0}\left(u_{0}\right)}} \leq C_{0}\|f\|_{L^{p_{0}}\left(v_{0}\right)}, \\
& \|S f\|_{L^{p_{1}\left(u_{1}\right)}} \leq C_{1}\|f\|_{L^{p_{1}}\left(v_{1}\right)} .
\end{aligned}
$$

Then,

$$
\|S f\|_{L^{p}(u)} \leq C\|f\|_{L^{p}(v)}
$$

holds for any $0<\theta<1$ and $1 / p=\theta / p_{0}+(1-\theta) / p_{1}$, where $u=u_{0}^{p \theta / p_{0}} u_{1}^{p(1-\theta) / p_{1}}, v=v_{0}^{p \theta / p_{0}} v_{1}^{p(1-\theta) / p_{1}}$, and $C \leq C_{0}^{\theta} C_{1}^{1-\theta}$.

Lemmas 10 and 11 are the main tools in proving Theorem 5.

\section{Proof of Theorem 5}

In this section, we will prove Theorem 5 by induction, which is partly motivated by $[8,10]$. We begin with the proof of $(1)$. For any nonzero real polynomial $P(x, y)$ in $x$ and $y$, there are $k, l, m \geq 0$ such that

$$
P(x, y)=a_{k l} x^{k} y^{l}+R(x, y)
$$

with $a_{k l} \neq 0$ and

$$
R(x, y)=\sum_{0 \leq \alpha<k, 0 \leq \beta \leq m} a_{\alpha \beta} x^{\alpha} y^{\beta}+\sum_{0 \leq \beta<l} a_{k \beta} x^{k} y^{\beta} .
$$

We will write $d_{x}(P)=k$ and $d_{y}(P)=l$. Below we will carry out the argument by using a double induction on $k$ and $l$.

If $d_{x}(P)=0$ and $d_{y}(P)$ is arbitrary, then $P(x, y)=P(y)$ and $T^{+} f$ can be written as

$$
T^{+} f(x)=\lim _{\varepsilon \rightarrow 0^{+}} \int_{x+\varepsilon}^{\infty} K(x-y) g(y) d y,
$$

where $g(y)=e^{i P(y)} f(y)$. Therefore, the conclusion of Theorem 5 follows from Theorem 4 .

Let $k \geq 1$ and assume that the conclusion of Theorem 5 holds for all $P(x, y)$ with $d_{x}(P) \leq k-1$ and $d_{y}(P)$ arbitrary.

We will now prove that the conclusion of Theorem 5 holds for all $P(x, y)$ with $d_{x}(P)=k$ and $d_{y}(P)$ arbitrary.

$$
\begin{aligned}
& \text { If } d_{x}(P)=k \text { and } d_{y}(P)=0 \text {, then } \\
& \qquad P(x, y)=a_{k 0} x^{k}+Q(x, y)
\end{aligned}
$$

with $d_{x}(Q) \leq k-1$. By taking the factor $e^{i a_{k 0} x^{k}}$ out of the integral sign, we see that this case follows from the above inductive hypothesis.

Suppose $l \geq 1$ and the desired bound holds when $d_{x}(P)=$ $k$ and $d_{y}(P) \leq l-1$.

Now, let $P(x, y)$ be a polynomial with $d_{x}(P)=k$ and $d_{y}(P)=l$, as given in (34)

Case $1\left(\left|a_{k l}\right|=1\right)$. Write

$$
\begin{aligned}
T^{+} f(x)= & \int_{x}^{1+x} e^{i P(x, y)} K(x-y) f(y) d y \\
& +\sum_{j=1}^{\infty} \int_{2^{j-1}+x}^{2^{j}+x} e^{i P(x, y)} K(x-y) f(y) d y \\
= & : T_{0}^{+} f(x)+\sum_{j=1}^{\infty} T_{j}^{+} f(x) .
\end{aligned}
$$

Take any $h \in \mathbb{R}$, and write

$$
P(x, y)=a_{k l}(x-h)^{k}(y-h)^{l}+R(x, y, h),
$$

where the polynomial $R(x, y, h)$ satisfies the induction assumption and the coefficients of $R(x, y, h)$ depend on $h$.

We consider first the estimates for $T_{0}^{+}$. It is easy to check that

$$
\begin{aligned}
T_{0}^{+} f(x) & \\
= & \int_{x}^{1+x} e^{i\left(R(x, y, h)+a_{k l}(y-h)^{k+l}\right)} K(x-y) f(y) d y \\
& +\int_{x}^{1+x}\left\{e^{i P(x, y)}-e^{i\left(R(x, y, h)+a_{k l}(y-h)^{k+l}\right)}\right\} \\
& \times K(x-y) f(y) d y \\
= & : T_{01}^{+} f(x)+T_{02}^{+} f(x) .
\end{aligned}
$$

Now we split $f$ into three parts as

$$
\begin{aligned}
f(y)= & f(y) \chi_{\{|y-h|<1 / 2\}}(y) \\
& +f(y) \chi_{\{1 / 2 \leq|y-h|<5 / 4\}}(y) \\
& +f(y) \chi_{\{|y-h| \geq 5 / 4\}}(y) \\
= & : f_{1}(y)+f_{2}(y)+f_{3}(y) .
\end{aligned}
$$

Observe that if $|x-h|<1 / 4$, then

$$
\begin{aligned}
& T_{01}^{+} f_{1}(x) \\
& =\int_{x}^{1+x} e^{i\left(R(x, y, h)+a_{k l}(y-h)^{k+l}\right)} K(x-y) f_{1}(y) d y .
\end{aligned}
$$


Thus, it follows from the induction assumption that

$$
\begin{aligned}
& \int_{|x-h|<1 / 4}\left|T_{01}^{+} f_{1}(x)\right|^{p} w(x) d x \\
& \leq C \int_{|y-h|<1 / 2}|f(y)|^{p} w(y) d y,
\end{aligned}
$$

where $C$ is independent of $h$ and the coefficients of $P(x, y)$.

Notice that if $|x-h|<1 / 4,1 / 2 \leq|y-h|<5 / 4$, then $y-x>1 / 4$. Thus,

$$
\begin{aligned}
\left|T_{01}^{+} f_{2}(x)\right| & \leq C \int_{x+1 / 4}^{x+1}\left|K(x-y) f_{2}(y)\right| d y \\
& \leq C M^{+}\left(f_{2}\right)(x) .
\end{aligned}
$$

So we have

$$
\begin{aligned}
& \int_{|x-h|<1 / 4}\left|T_{01}^{+} f_{2}(x)\right|^{p} w(x) d x \\
& \leq C \int_{|y-h|<5 / 4}|f(y)|^{p} w(y) d y,
\end{aligned}
$$

where $C$ is independent of $h$ and the coefficients of $P(x, y)$.

Again observe that if $|x-h|<1 / 4$ and $|y-h| \geq 5 / 4$, then $y-x>1$. Thus,

$$
T_{01}^{+} f_{3}(x)=0 .
$$

Combining (43), (45), and (46), we get

$$
\begin{aligned}
& \int_{|x-h|<1 / 4}\left|T_{01}^{+} f(x)\right|^{p} w(x) d x \\
& \leq C \int_{|y-h|<5 / 4}|f(y)|^{p} w(y) d y,
\end{aligned}
$$

where $C$ is independent of $h$ and the coefficients of $P(x, y)$.

Evidently, if $|x-h|<1 / 4$ and $0<y-x<1$, then

$$
\begin{aligned}
& \left|e^{i P(x, y)}-e^{i\left(R(x, y, h)+a_{k l}(y-h)^{k+l}\right)}\right| \\
& \leq C\left|a_{k l}\right||x-y|=C(y-x) .
\end{aligned}
$$

Therefore, when $|x-h|<1 / 4$, we have

$$
\begin{aligned}
\left|T_{02}^{+} f(x)\right| & \leq C \int_{x}^{x+1}|f(y)| d x \\
& \leq C M^{+}\left(f(\cdot) \chi_{B(h, 5 / 4)}(\cdot)\right)(x) .
\end{aligned}
$$

It follows from Theorem 3 that

$$
\begin{aligned}
& \int_{|x-h|<1 / 4}\left|T_{02}^{+} f(x)\right|^{p} w(x) d x \\
& \leq C \int_{|y-h|<5 / 4}|f(y)|^{p} w(y) d y,
\end{aligned}
$$

where $C$ is independent of $h$ and the coefficients of $P(x, y)$.
From (47) and (50), it follows that the inequality

$$
\begin{aligned}
& \int_{|x-h|<1 / 4}\left|T_{0}^{+} f(x)\right|^{p} w(x) d x \\
& \quad \leq C \int_{|y-h|<5 / 4}|f(y)|^{p} w(y) d y
\end{aligned}
$$

holds uniformly in $h \in \mathbb{R}^{+}$, which implies

$$
\left\|T_{0}^{+} f\right\|_{L^{p}(w)} \leq C\|f\|_{L^{p}(w)}
$$

where $C$ is independent of the coefficients of $P(x, y)$ and $w \in$ $A_{p}^{+}$.

We proceed with the proof of Theorem 5 with the estimates for $T_{j}^{+} f$. Because of the size condition (2), we observe that for $j \geq 1$

$$
\left|T_{j}^{+} f(x)\right| \leq \int_{2^{j-1}+x}^{2^{j}+x} \frac{|f(y)|}{|x-y|} d y \leq \operatorname{CM}^{+}(f)(x),
$$

where $C$ is independent of $j$. By Lemma 10, we know that there exists $\varepsilon>0$ such that $w^{1+\varepsilon} \in A_{p}^{+}$. Thus we have

$$
\left\|T_{j}^{+} f\right\|_{L^{p}\left(w^{1+\varepsilon}\right)} \leq C\|f\|_{L^{p}\left(w^{1+\varepsilon}\right)},
$$

where $C$ is independent of $j$. We now only need to recall Lemma 3.7 in [16] to see that

$$
\left\|T_{j}^{+} f\right\|_{L^{p}} \leq C 2^{-j \delta}\|f\|_{L^{p}},
$$

where $C$ depends only on the total degree of $P(x, y)$ and $\delta>$ 0 . It follows from (54), (55), and Lemma 11 that

$$
\left\|T_{j}^{+} f\right\|_{L^{p}(w)} \leq C 2^{-j \theta \delta}\|f\|_{L^{p}(w)},
$$

where $0<\theta<1, \theta$ is independent of $j$, and $C$ depends only on the total degree of $P(x, y)$.

From (52) and (56), it is clear that when $w \in A_{p}^{+}$,

$$
\left\|T^{+} f\right\|_{L^{p}(w)} \leq C\|f\|_{L^{p}(w)},
$$

where $C$ depends only on the total degree of $P(x, y)$.

Case $2\left(\left|a_{k l}\right| \neq 1\right)$. In this case, we write $\lambda=\left|a_{k l}\right|^{1 /(k+l)}$ and

$$
\begin{aligned}
P(x, y) & =\lambda^{-(k+l)} a_{k l}(\lambda x)^{k}(\lambda y)^{l}+R\left(\frac{\lambda x}{\lambda}, \frac{\lambda y}{\lambda}\right) \\
& =Q(\lambda x, \lambda y) .
\end{aligned}
$$

Therefore,

$$
\begin{aligned}
T^{+} f(x) & =\text { p.v. } \int e^{i \mathrm{Q}(\lambda x, \lambda y)} K(x-y) f(y) d y \\
& =\text { p.v. } \int e^{i Q(\lambda x, y)} K\left(\frac{\lambda x}{\lambda}-\frac{y}{\lambda}\right) f\left(\frac{y}{\lambda}\right) \lambda^{-1} d y \\
& =T_{\lambda}^{+}\left(f\left(\frac{\dot{\lambda}}{\lambda}\right)\right)(\lambda x)
\end{aligned}
$$


where $K_{\lambda}(x-y)=\lambda^{-1} K(x / \lambda-y / \lambda)$ and

$$
T_{\lambda}^{+} f(x)=\text { p.v. } \int e^{i Q(x, y)} K_{\lambda}(x-y) f(y) d y .
$$

It is easy to check that $K_{\lambda}$ satisfies (2) and (10). We have thus established that

$$
\left\|T_{\lambda}^{+} f\right\|_{L^{p}(w)} \leq C\|f\|_{L^{p}(w)}
$$

with similar statements as in Case 1. By Lemma 7, we have

$$
\begin{aligned}
\int \mid T^{+} & \left.f(x)\right|^{p} w(x) d x \\
& =\int\left|T_{\lambda}^{+} f\left(\frac{\cdot}{\lambda}\right)(\lambda x)\right|^{p} w(x) d x \\
& =\lambda^{-1} \int\left|T_{\lambda}^{+} f\left(\frac{\cdot}{\lambda}\right)(x)\right|^{p} w\left(\frac{x}{\lambda}\right) d x \\
& \leq C \int\left|f\left(\frac{x}{\lambda}\right)\right|^{p} w\left(\frac{x}{\lambda}\right) d x \\
& =C \int|f(x)|^{p} w(x) d x ;
\end{aligned}
$$

that is,

$$
\left\|T^{+} f\right\|_{L^{p}(w)} \leq C\|f\|_{L^{p}(w)},
$$

where $C$ depends on the total degree of $P(x, y)$ but not on the coefficients of $P(x, y)$.

(2) We omit the details, since they are very similar to that of the proof of (1) with $w \in A_{p}^{-}$instead of $w \in A_{p}^{+}$.

\section{Conflict of Interests}

The authors declare that there is no conflict of interests regarding the publication of this paper.

\section{Acknowledgments}

This work was partially supported by NSF of China (Grant nos. 11271175, 10931001, and 11301249), NSF of Shandong Province (Grant no. ZR2012AQ026), the AMEP and DYSP of Linyi University, and the Key Laboratory of Mathematics and Complex System (Beijing Normal University), Ministry of Education, China.

\section{References}

[1] E. M. Stein, Harmonic Analysis (Real-variable methods, orthogonality, and oscillatory integrals), vol. 43 of Princeton Mathematical Series, Princeton University Press, Princeton, NJ, USA, 1993.

[2] L. Grafakos, Classical and Modern Fourier Analysis, Pearson Education, Upper Saddle River, NJ, USA, 2004.

[3] Y. Hu and Y. Pan, "Boundedness of oscillatory singular integrals on Hardy spaces," Arkiv för Matematik, vol. 30, no. 2, pp. 311320, 1992.
[4] S. Lu, "Multilinear oscillatory integrals with Calderón-Zygmund kernel," Science in China A, vol. 42, no. 10, pp. 1039-1046, 1999.

[5] S. Lu and Y. Zhang, "Criterion on $L^{p}$-boundedness for a class of oscillatory singular integrals with rough kernels," Revista Matemática Iberoamericana, vol. 8, no. 2, pp. 201-219, 1992.

[6] Y. Pan, "Hardy spaces and oscillatory singular integrals," Revista Matemática Iberoamericana, vol. 7, no. 1, pp. 55-64, 1991.

[7] D. H. Phong and E. M. Stein, "Singular integrals related to the Radon transform and boundary value problems," Proceedings of the National Academy of Sciences of the United States of America, vol. 80, no. 24, pp. 7697-7701, 1983.

[8] F. Ricci and E. M. Stein, "Harmonic analysis on nilpotent groups and singular integrals. I. Oscillatory integrals," Journal of Functional Analysis, vol. 73, no. 1, pp. 179-194, 1987.

[9] R. R. Coifman and C. Fefferman, "Weighted norm inequalities for maximal functions and singular integrals," Studia Mathematica, vol. 51, pp. 241-250, 1974.

[10] S. Lu and Y. Zhang, "Weighted norm inequality of a class of oscillatory singular operators," Chinese Science Bulletin, vol. 37, pp. 9-13, 1992.

[11] E. Sawyer, "Weighted inequalities for the one-sided HardyLittlewood maximal functions," Transactions of the American Mathematical Society, vol. 297, no. 1, pp. 53-61, 1986.

[12] F. J. Martín-Reyes, P. Ortega Salvador, and A. de la Torre, "Weighted inequalities for one-sided maximal functions," Transactions of the American Mathematical Society, vol. 319, no. 2, pp. 517-534, 1990.

[13] F. J. Martín-Reyes, L. Pick, and A. de la Torre, " $A_{\infty}^{+}$condition," Canadian Journal of Mathematics, vol. 45, no. 6, pp. 1231-1244, 1993.

[14] F. J. Martín-Reyes and A. de la Torre, "One-sided BMO spaces," Journal of the London Mathematical Society, vol. 49, no. 3, pp. 529-542, 1994.

[15] H. Aimar, L. Forzani, and F. J. Martín-Reyes, "On weighted inequalities for singular integrals," Proceedings of the American Mathematical Society, vol. 125, no. 7, pp. 2057-2064, 1997.

[16] Z. Fu, S. Lu, S. Sato, and S. Shi, "On weighted weak type norm inequalities for one-sided oscillatory singular integrals," Studia Mathematica, vol. 207, no. 2, pp. 137-151, 2011.

[17] F. J. Martín-Reyes, P. Ortega Salvador, and A. de la Torre, "Weights for one-sided operators," in Recent Development in Real and Harmonic Analysis, Applied and Numerical Harmonic Analysis, Birkhäuser, Boston, Mass, USA, 2009.

[18] M. S. Riveros and A. de la Torre, "On the best ranges for $A_{p}^{+}$ and $\mathrm{RH}_{r}^{+}$, Czechoslovak Mathematical Journal, vol. 51, no. 2, pp. 285-301, 2001.

[19] F. J. Martín-Reyes, "New proofs of weighted inequalities for the one-sided Hardy-Littlewood maximal functions," Proceedings of the American Mathematical Society, vol. 117, no. 3, pp. 691-698, 1993.

[20] E. M. Stein and G. Weiss, "Interpolation of operators with change of measures," Transactions of the American Mathematical Society, vol. 87, pp. 159-172, 1958. 


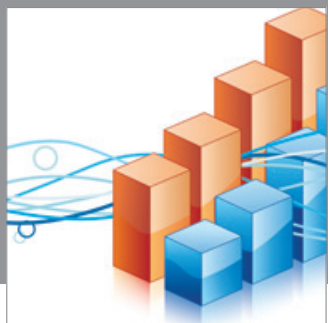

Advances in

Operations Research

mansans

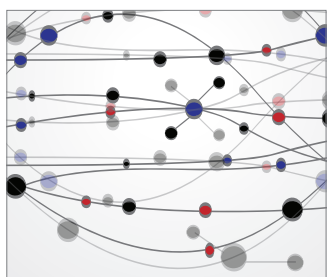

The Scientific World Journal
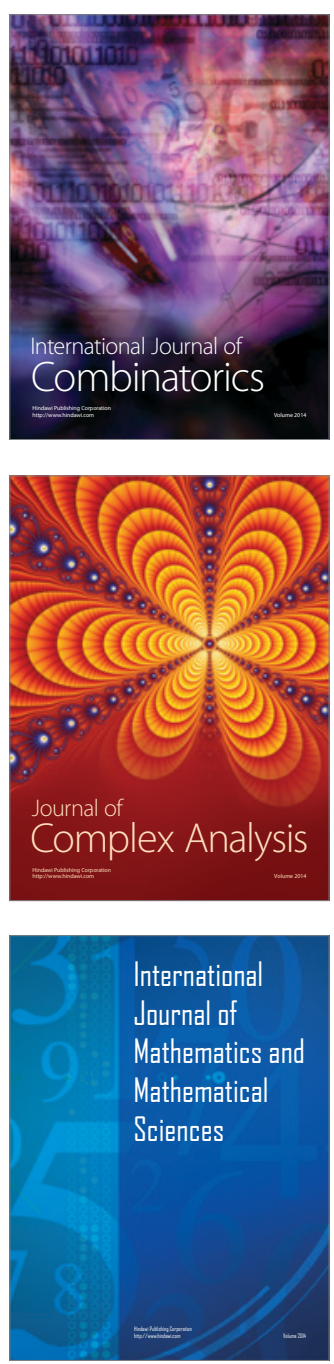
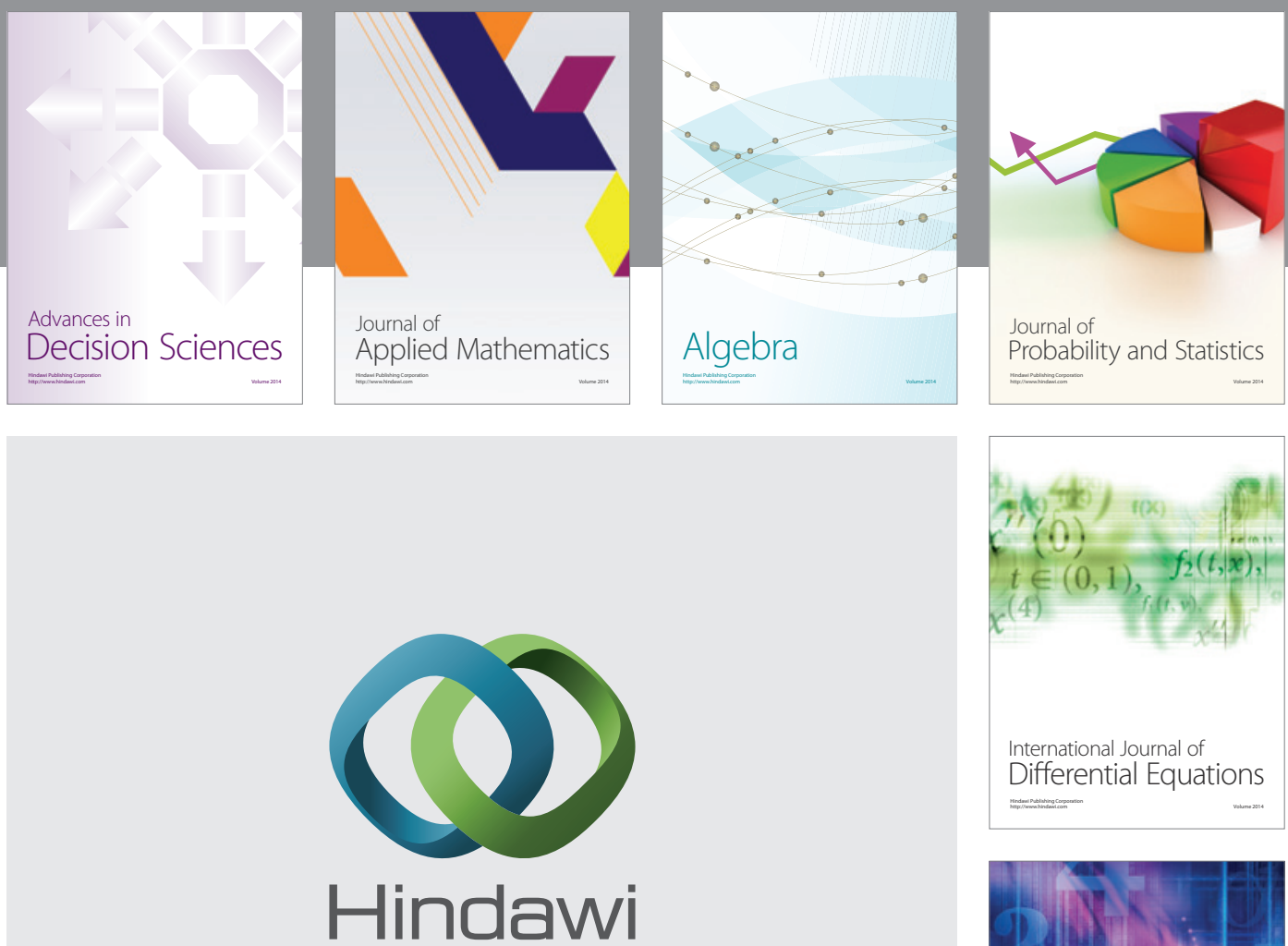

Submit your manuscripts at http://www.hindawi.com
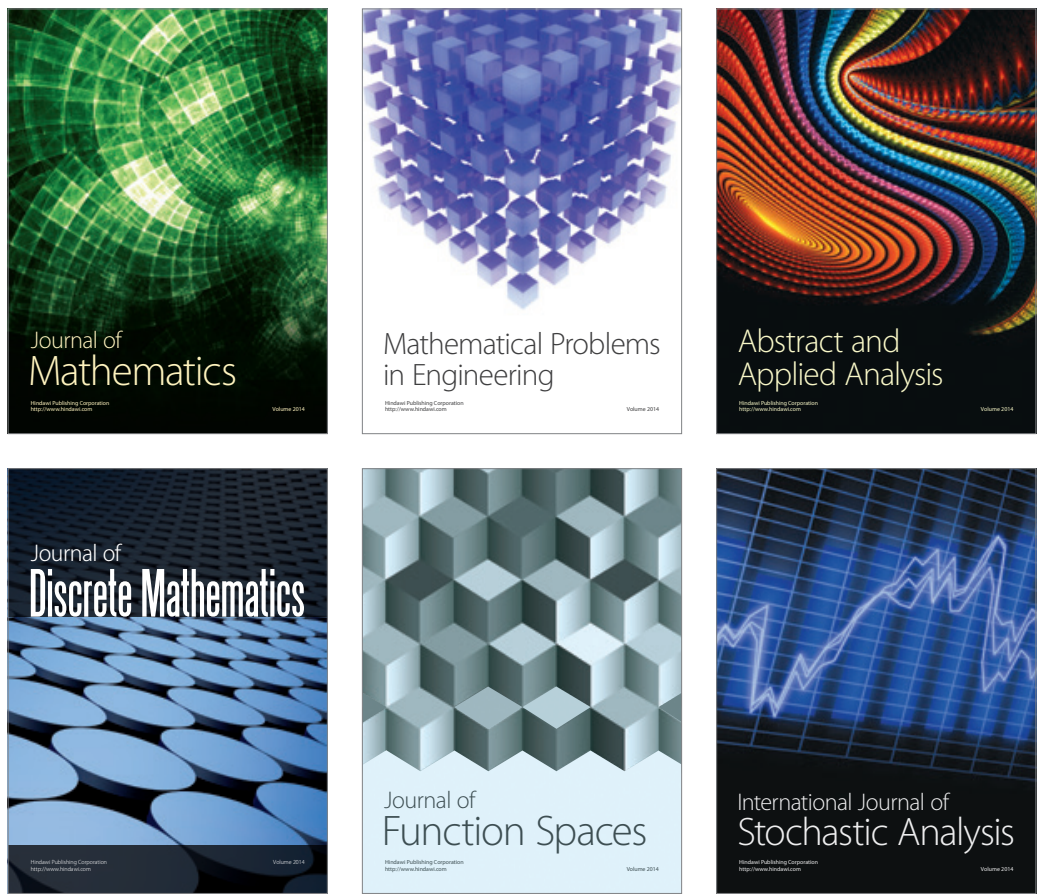

Journal of

Function Spaces

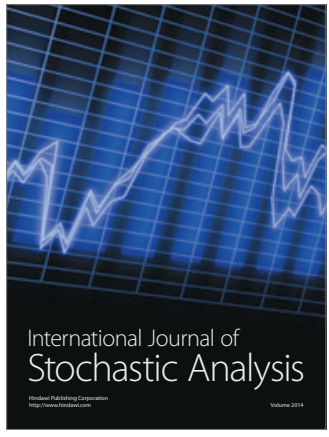

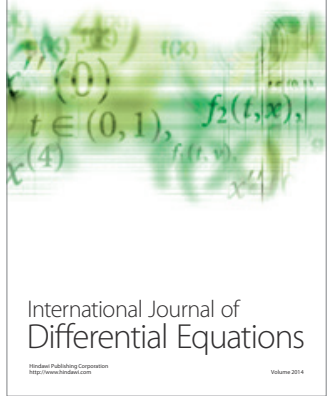
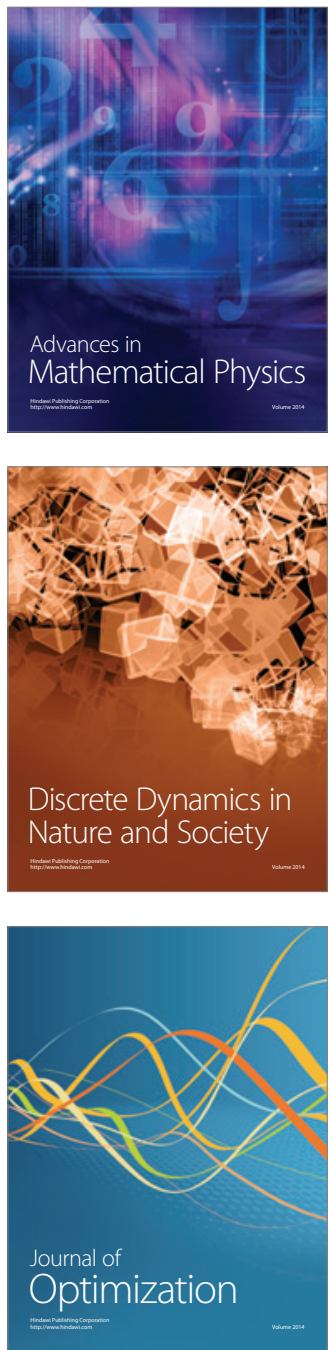